$$
\text { CONF- } 970396--1
$$

Note: This is a preprint of a paper being submitted for publication. Contents of this paper should not be quoted nor referred to without permission of the author(s).

\title{
Low-Cost Metal Substrates for Films with Aligned Grain Structures
}

D. P. Norton, J. D. Budai, A. Goyal, D. H. Lowndes, D. M. Kroeger, D. K. Christen, M. Paranthaman, and E. D. Specht

\author{
Submitted to: \\ First Conference on \\ Future Generation \\ Photovoltaic \\ Technologies \\ March 24-26, 1997 \\ Denver, Colorado
}

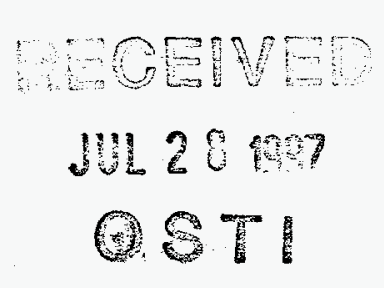

"The submitted manuscript has been authored by a contractor of the U.S. Government under contract DE-AC05-960R22464. Accordingly, the U.S. Government retains a nonexclusive, royalty-free license to publish or reproduce the published form of this contribution, or allow others to do so, for U.S. Government purposes."

Prepared by

Solid State Division

Oak Ridge National Laboratory

P.O. Box 2008

Oak Ridge, Tennessee 37831-6056

managed by

LOCKHEED MARTIN ENERGY RESEARCH CORP.

for the

U.S. DEPARTMENT OF ENERGY

under contract DE-AC05-96OR22464 


\title{
Low-Cost Metal Substrates for Films with Aligned Grain Structures
}

\author{
D. P. Norton, J. D. Budai, A. Goyal, D. H. Lowndes, D. M. Kroeger, \\ D. K. Christen, M. Paranthaman, and E. D. Specht
}

Oak Ridge National Laboratory, Oak Ridge, TN 37831-6056

\begin{abstract}
Polycrystalline metal substrates that possess a significant amount of in-plane and out-of-plane crystallographic texture have recently been developed for high-temperature superconducting film applications. These substrates enable the virtual elimination of large angle grain boundaries in subsequent epitaxial films, having been successfully utilized in various oxide thin film architectures. This paper describes the characteristics of these substrates, and briefly discusses their potential applicability in polycrystalline thin-film photovoltaic applications.
\end{abstract}

\section{INTRODUCTION}

For many thin-film device applications, substrate selection is a primary determinant of film characteristics and device performance, as well as a significant cost factor. As such, significant attention has been given to device structures fabricated on a variety of substrate materials, including inexpensive glass, polycrystalline ceramics and metals, and single crystals. Of course, films deposited on amorphous or polycrystalline substrates are, at best, polycrystalline with a random distribution of grain boundaries that can impose serious limitations on the performance of electronic devices (1). Extended defects at the boundary result in dangling bonds that introduce electronic states in the band gap of semiconductors and insulators. This typically leads to a reduction in both the carrier lifetime and mobility. Grain boundaries also introduce an electronic interface between grains, with grain boundary charging due to permanently trapped charges at the interface. Additional difficulties associated with grain boundaries include impurity segregation and secondary phase formation at the boundary. In many instances, elimination of grain boundaries with the use of 
epitaxial films grown on single crystal substrates results in superior device performance. Epitaxial films reproduce the crystallinity from the substrate, resulting in a single crystal-like film of the desired material. Unfortunately, the cost of single crystal substrates, as well as limitations on the available size, significantly limits the applicability of single crystals in the elimination of grain boundaries in electronic materials for large-area applications.

\section{GRAIN ALIGNMENT WITH BIAXIALLY-TEXTURED SUBSTRATES}

Until recently, the manipulation of grain boundaries through a significant reduction of the misorientation angle between adjacent grains has been limited to cases where single crystal substrates could used. However, recent developments in the area of superconductivity have made possible the formation of large-area substrates in which the crystallographic orientation at the substrate surface is highly textured, with only low angle grain boundaries present in the material. When considering the properties of polycrystalline high temperature superconducting oxides, large-angle grain boundaries have a profound and detrimental effect on the ability of these materials to carry significant electrical currents, as indicated by the superconducting critical current density, $\mathrm{J}_{\mathrm{c}}$. Key experiments in which the critical current density was measured across single grain boundaries in $\mathrm{YBa}_{2} \mathrm{Cu}_{3} \mathrm{O}_{7}$ (YBCO) films deposited on $\mathrm{SrTiO}_{3}$ bicrystals clearly indicate that grain boundaries greater than $10^{\circ}$ dramatically reduce $J_{c}$. This observation motivated an effort to develop a means for achieving crystallographic alignment with the virtual elimination of large angle grain boundaries in long-length substrates.

Two different approaches have emerged that are effective in producing a significant degree of in-plane and out-of-plane crystallographic texture in largearea, long-length substrates. One technique involves the use of energetic ions directed at the surface of a growing film to induce crystallographic orientation of the depositing film (2). This technique, known as ion beam-assisted deposition (IBAD), results in highly textured films, with particular success achieved with yttria-stabilized zirconia (YSZ) buffer layers for superconducting oxide film growth on nickel-based alloys. Similar approaches using energetic ions in magnetron sputtering and pulsed-laser deposition have also been developed $(3,4)$. In each case, the impinging energetic ions create crystallographic texture, either through an ion channeling or preferential sputtering mechanism. A competing approach, designated as rolling assisted biaxially-textured substrates (RABiTS), results in a crystallographically textured substrate by thermomechnical deformation of a metal (2). Both the IBAD and RABiTS approach have led to 
significant enhancements in the critical current density for deposited YBCO films when compared to randomly oriented HTS materials.

\section{The RABiTS Concept}

The basic concept of RABiTS, schematically illustrated in Fig. 1, begins with the formation of crystallographic texture in a metal foil by thermomechanical deformation. It is well known that significant in-plane and out-of-plane texture can be induced in most metals simply by rolling and annealing (6). In some cases, a single component of texture can be achieved, with the foil emulating a poor single crystal with a broad mosaic spread in the texture. A good example of this is coldrolled and annealed nickel. Figure 2 shows the out-of-plane and in-plane texture as determined by four circle $\mathrm{x}$-ray diffraction, in a pure nickel foil that is formed by rolling at room temperature and annealing in a reducing atmosphere. The diffraction pattern shows significant in-plane and out-of-plane texture, with the full width at half maximum (FWHM) of the in-plane and out-of-plane diffraction peaks approximately $7-10^{\circ}$. The grain size of the nickel foil is on the order of 50$100 \mu \mathrm{m}$.

\section{RABiTS Process}

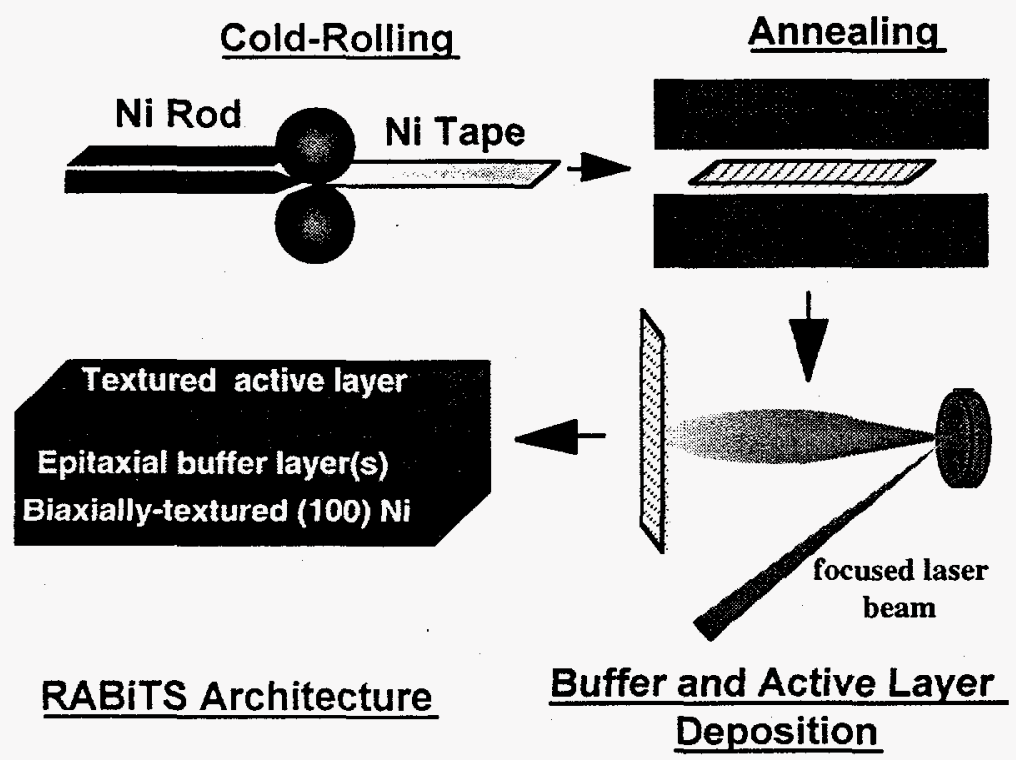

FIGURE 1. The RABITS process for fabricating biaxially-textured substrates for polycrystalline film growth. Crystallographic alignment in the substrate can virtually eliminate large-angle grain boundaries in subsequent epitaxial films. 

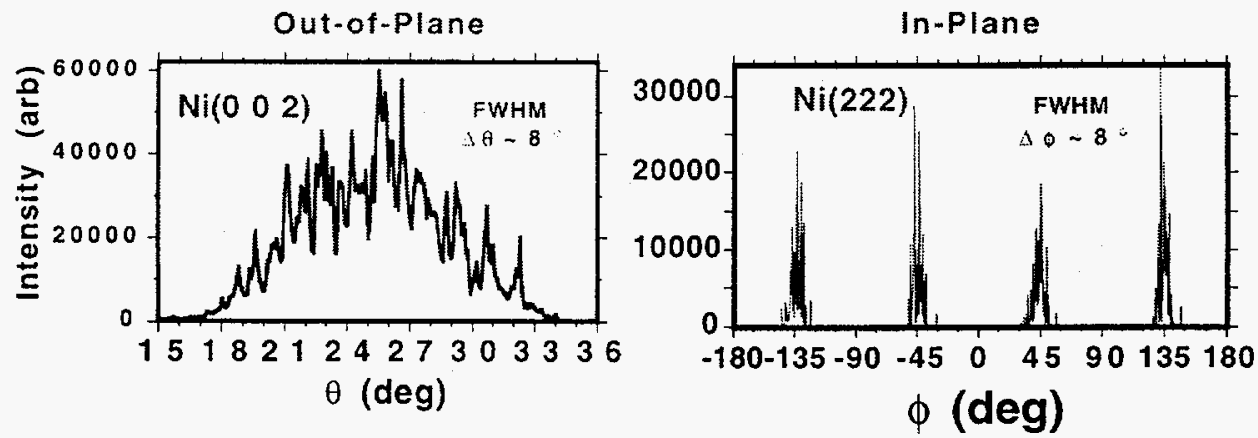

FIGURE 2. Significant in-plane and out-of-plane crystallographic texture can be achieved in pure nickel by simply cold-rolling and annealing. This figure shows the $\mathrm{x}$-ray diffraction $\theta$-scan through the $\mathrm{Ni}(200)$, as well as the $\phi$-scan through the $\mathrm{Ni}(220)$

In most cases, the epitaxial growth of electronic materials of interest directly on a metal is not feasible due to chemical interaction of the film with the substrate. An epitaxial buffer layer that chemically isolates the active layer from the metal substrate while transferring the texture from the substrate to the film will be needed. Previous work on HTS conductors based on the RABiTS approach has implemented various oxide buffer layers to separate the superconducting film from a nickel foil. Figure 3 shows a schematic illustration, along with the $\mathrm{x}$-ray diffraction scans, for a $\mathrm{YBCO} / \mathrm{YSZ} / \mathrm{CeO}_{2} / \mathrm{Ni}$ structure grown by pulsed-laser deposition (PLD), in which the $\mathrm{YSZ} / \mathrm{CeO}_{2}$ buffer layers isolate the nickel substrate from the HTS film. Typically, the epitaxial growth of a (001)oriented cubic oxide on a (001) $\mathrm{Ni}$ surface is inhibited by the formation of (111) $\mathrm{NiO}$ at the oxide/metal interface. The $\mathrm{Ni}$ substrates were annealed at $900^{\circ} \mathrm{C}$ in a $4 \% \mathrm{H}_{2} / \mathrm{Ar}$ gas mixture prior to film growth to reduce any $\mathrm{NiO}$ on the substrate surface. In order to further suppress the formation of $\mathrm{NiO}$ and achieve (001)oriented epitaxy directly on the (001) Ni surface, $\mathrm{H}_{2}$ gas was introduced into the PLD chamber during the initial stages of $\mathrm{CeO}_{2}$ growth. Hydrogen is effective in reducing $\mathrm{NiO}$, while having little effect on the $\mathrm{CeO}_{2}$ film. This (001)-oriented $\mathrm{CeO}_{2}$ layer provides an oxide template directly on the metal surface for the subsequent epitaxial growth of additional oxide buffer and HTS layers. The inplane $\phi$-scans and out-of-plane rocking curves show that the crystallographic texture in the nickel foil is replicated in each of the layers, including the superconducting YBCO film. The in-plane FWHM for all of the layers is $\sim 6.8^{\circ}$, indicating excellent epitaxy of the oxide layers with the biaxially textured metal. If the grain-to-grain misorientation angles are uncorrelated with a normal distribution, $\sim 90 \%$ of the $\mathrm{Ni}$ grains have in-plane misorientation angles of $7^{\circ}$ or less. 

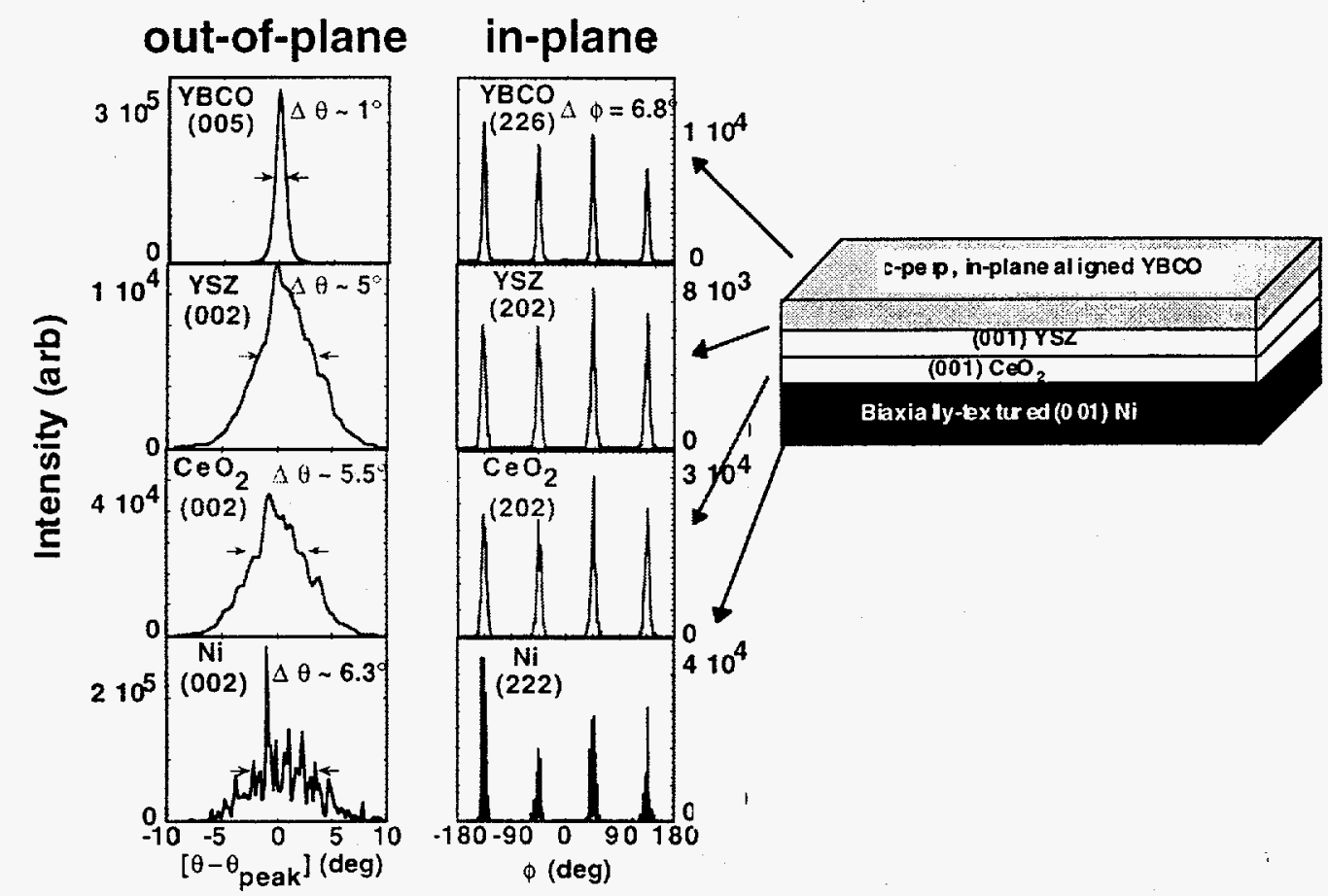

FIGURE 3. X-ray diffraction rocking curves and $\phi$-scans showing the in-plane and outof-plane texture in a multilayer structure for YBCO growth on rolled-textured nickel. Similar architectures would be required for other applications of textured metals as substrates.

\section{Biaxially-Textured Substrates for Photovoltaics?}

With the successful implementation of biaxially-textured substrates for long-length HTS applications, one can begin to consider the applicability of this approach in other thin-film applications, including photovoltaics. Challenges associated with using rolled-textured metal substrates for photovoltaic applications are numerous. The specific rolling-induced texture is highly dependent on material. Identification of an attractive substrate material that possesses a reasonable match in lattice parameter and thermal expansion coefficient, and can be rolled-textured with a useful texture component may prove difficult. In addition, the typical dislocation densities in rolled-textured metals are orders of magnitude larger than that generally required for semiconductor solar cell material. This will require a complex, multilayer buffer layer architecture that not only chemically isolates the semiconductor film from the metal substrate, but also effectively terminates dislocations. With the RABiTS approach, the polycrystalline substrates possess relatively large grain sizes, thus proving 
attractive for film growth when large grain polycrystalline material is advantageous regardless of the grain boundary misorientation. However, the most fundamental issue that must be addressed in determining the potential benefit of using any biaxially-textured substrate for photovoltaics involves understanding the role of grain boundary misorientation in limiting the solar cell efficiency for photovoltaics. Previous work in this area has primarily focused on $\mathrm{Ge}$ grain boundaries (8), with less work reported on $\mathrm{Si}(9), \mathrm{GaAs}(10)$, and other compound semiconductors. For materials with high sensitivity to low or moderate densities of dislocations, one would anticipate that almost any grain boundary would significantly reduce carrier lifetime and solar cell efficiency. However, there are few studies in which the specific electronic behavior of grain boundaries are determined as the misorientation angle is varied for small angles $\left(<10^{\circ}\right)$, including the role of misorientation on defect passivation. For many photovoltaic materials considered candidates for thin-film applications, the effects of dislocations and grain boundaries on semiconductor properties is less clear. Obviously, a better understanding of how relevant semiconductor properties vary with grain boundary misorientation is needed in evaluating the potential benefits of textured substrates in photovoltaics

\section{ACKNOWLEDGMENTS}

This research was sponsored by ORNL, managed by Lockheed Martin Energy Research Corporation, for the U.S. Department of Energy Office of Energy Research and the Office of Energy Efficiency and Renewable Energy, under contract DE-AC0596OR22464.

\section{REFERENCES}

1. L. L. Kazmerski, in Polycrystalline and Amorphous Thin Films and Devices, New York: Academic Press, 1980, ch. 3, pp. 59-133.

2. Y. Iijima, N. Tanabe, O. Kohno, Y. Ikeno, Appl. Phys. Lett. 60, 769 (1992); R. P. Reade, P. Berdahl, R. E. Russo, S. M. Garrison, Appl. Phys. Lett. 61, 2231 (1992).

3. M. Fukutomi, S. Aoki, K. Komori, R. Chatterjee, H. Maeda, Physica $C$ 219, 333 (1994).

4. K. Hasegawa, N. Yoshida, K. Fujino, H. Mukai, K. Hayashi, K. Sato, T. Ohkuma, S. Honjyo, H. Ishii, and T. Hara, in Proceedings of the 1996 International Cryogenics Materials Conference (in press).

5. D. P. Norton, et al., Science 274, 755 (1996); A. Goyal, et al., Appl. Phys. Lett. 69, 1795 (1996). 
6. H. Makita, S. Hanada, O. Izumi, Acta Metall. 36, 403 (1988).

7. N. N. Khoi, W. W. Smeltzer, J. D. Embury, J. Electrochem. Soc. 122, 1495 (1975).

8. B. Reed, O. A. Weinreich, and H. F. Matere, Phys. Rev. 113, 454 (1959); A. G. Tweet, Phys. Rev. 99, 1182 (1955); R. K. Mueller, J. Phys. Chem. Solids 8, 157 (1959).

9. Y. Matukura, J. Phys. Soc. Japan 16, 842 (1961).

10. J. P. Salerno, J. C. C. Fan, R. W. McClelland, P. Vohl, J. G. Mavroides, and C. O. Bozler, "Electronic Properties of Grain Boundaries in GaAs: A Study of Oriented Bicrystals Prepared By Epitaxial Lateral Overgrowth," Technical Report 669,

Technology (1984).

\section{DISCLAIMER}

This report was prepared as an account of work sponsored by an agency of the United States Government. Neither the United States Government nor any agency thereof, nor any of their empleyees, makes any warranty, express or implied, or assumes any legal liability or responsibility for the accuracy, completeness, or usefulness of any information, apparatus, product, or process disclosed, or represents that its use would not infringe privately owned rights. Reference herein to any specific commercial product, process, or service by trade name, trademark, manufacturer, or otherwise does not necessarily constitute or imply its endorsement, recom: mendation, or favoring by the United States Government or any agency thereof. The views and opinions of authors expressed herein do not necessarily state or reflect those of the United States Government or any agency thereof. 


\section{DISCLAMMER}

Portions of this document may be illegible in electronic image products. Images are produced from the best available original docoment. 